\title{
A moving-mesh relaxation scheme for one-dimensional barotropic two-phase flow with cavitation *
}

\author{
Keh-Ming Shyue \\ Department of Mathematics, National Taiwan University, \\ Taipei 10617, Taiwan. \\ E-mail:shyue@math.ntu.edu.tw
}

\section{Introduction}

Cavitation is commonly defined as a phenomenon in a liquid-flowing system when the pressure of the liquid falls sufficiently low in some region of the flow so that vapor bubbles are formed. The study of the dynamics of cavitation is an active research in many fields of science and engineering. Typical examples in relation to various features and characteristics of cavitating flows can be found, for example, at the website: http://en.wikipedia.org/wiki/Cavitation, and references therein.

To compute cavitating flow numerically, one popular approach is to use a barotropic two-phase model (cf. [?]) in that, if we ignore the physical effects such as mass transfer, surface tension, and viscosity, the Eulerian formulation of the basic conservation laws in one space dimension takes the form

$$
\frac{\partial}{\partial t}\left(\begin{array}{c}
\alpha_{1} \rho_{1} \\
\alpha_{2} \rho_{2} \\
\rho u
\end{array}\right)+\frac{\partial}{\partial x}\left(\begin{array}{c}
\alpha_{1} \rho_{1} u \\
\alpha_{2} \rho_{2} u \\
\rho u^{2}+p
\end{array}\right)=0
$$

Here $\alpha_{k}, \rho_{k}$, and $p_{k}$ are the volume fraction, density, and pressure for the fluid phase $k, k=1,2$, respectively. The notations $\rho=\alpha_{1} \rho_{1}+\alpha_{2} \rho_{2}$, $p=\alpha_{1} p_{1}+\alpha_{2} p_{2}$, and $u$ denote in turn the total density, pressure, and particle velocity of the flow.

To close the system, the phasic pressure $p_{k}(\rho)$ is assumed to be a one-to-one function of the density (this should be true locally at the least), and so we may use the saturation condition $\alpha_{1}+\alpha_{2}=1$ directly, yielding a nonlinear algebraic equation to be solved for the pressure $p$,

$$
g(p)=\frac{\alpha_{1} \rho_{1}}{\rho_{1}(p)}+\frac{\alpha_{2} \rho_{2}}{\rho_{2}(p)}-1=0
$$

\footnotetext{
* The author was supported in part by National Science Council of Taiwan Grant \#96-2115-M-002-008-MY3.
} 
where $\alpha_{1} \rho_{1}$ and $\alpha_{2} \rho_{2}$ are known a priori. With that, there is no difficulty to compute the remaining flow variables such as $\rho_{1}, \rho_{2}, \alpha_{1}$, and $\alpha_{2}$.

In this case, it is known that combining (??) with (??) gives a hyperbolic model that is viable for a class of barotropic homogeneous twophase flow problems with and without cavitation. However, due to the non-monotonic behavior of the mixture sound speed (denoted by $c$ ) versus the volume fraction, $1 / \rho c^{2}=\alpha_{1} / \rho_{1} c_{1}^{2}+\alpha_{2} / \rho_{2} c_{2}^{2}$, it poses a major difficulty to maintain a suitable stability condition when the model is discretized by an explicit diffuse-interface type method.

To overcome this numerical difficulty, we are interested in a relaxation approach proposed by Saurel et al. [?] in that in addition to (??) a transport equation with a stiff relaxation source term is included in the model for the volume fraction, such as $\alpha_{1}$, of the form

$$
\frac{\partial \alpha_{1}}{\partial t}+u \frac{\partial \alpha_{1}}{\partial x}=\mu\left(p_{1}\left(\rho_{1}\right)-p_{2}\left(\rho_{2}\right)\right)
$$

where $\mu$ is a relaxation parameter. In contrast with the aforementioned conventional model, here the equilibrium pressure $p$ is obtained by taking the limit of infinite relaxation $\mu \rightarrow \infty$ to the solution of (??), yielding $p=p_{1}\left(\rho_{1}\right)=p_{2}\left(\rho_{2}\right)$, and so an algebraic equation for the relaxed volume fraction $\alpha_{1}$,

$$
g\left(\alpha_{1}\right)=p_{1}\left(\frac{\alpha_{1} \rho_{1}}{\alpha_{1}}\right)-p_{2}\left(\frac{\alpha_{2} \rho_{2}}{1-\alpha_{1}}\right)=0 .
$$

It is important to note that since this relaxation model will be solved by a fractional-step method, see Section ??, in the zero relaxation limit $\mu \rightarrow 0$, it possesses a nice monotonic behavior of the frozen speed of sound versus the mass fractions, $c_{f}^{2}=Y_{1} c_{1}^{2}+Y_{2} c_{2}^{2}$, and so is an easier one to use as compared to the conventional model for numerical approximation. Here $Y_{k}=\rho_{k} / \rho$ is the mass fraction for the fluid phase $k$.

Our goal in this work is to employ a state-of-the-art moving-mesh method for the efficient numerical resolution of this relaxation model for barotropic two-phase flow with cavitation in one space dimension. This is a fundamental step in our further development of the method to problems in multiple dimensions and also more complicated cavitating flows of practical importance (cf. [?, ?, ?]).

\section{Relaxation model in moving coordinates}

For the ease of the latter discussion, it is useful to write the relaxation model described above into a more compact expression by

$$
\frac{\partial q}{\partial t}+\frac{\partial f(q)}{\partial x}+B \frac{\partial q}{\partial x}=\mu \psi(q)
$$


where

$$
\begin{aligned}
q & =\left(\alpha_{1} \rho_{1}, \alpha_{2} \rho_{2}, \rho u, \alpha_{1}\right)^{T}, \\
f & =\left(\alpha_{1} \rho_{1} u, \alpha_{2} \rho_{2} u, \rho u^{2}+p, 0\right)^{T}, \\
B & =\operatorname{diag}(0,0,0, u) \\
\psi & =\operatorname{diag}\left(0,0,0, p_{1}\left(\rho_{1}\right)-p_{2}\left(\rho_{2}\right)\right) .
\end{aligned}
$$

Then, as a preliminary, we introduce a coordinate mapping from the physical domain $(t, x)$ to the computational domain $(\tau, \xi)$ as

$$
d t=d \tau, \quad d x=v d \tau+J d \xi
$$

where $v=\partial x / \partial \tau$ is the mesh velocity, and $J=\partial x / \partial \xi$ is the metric of the mapping. With that, (??) can be transformed into

$$
J \frac{\partial q}{\partial \tau}+\frac{\partial f(q)}{\partial \xi}+(B-v I) \frac{\partial q}{\partial \xi}=J \mu \psi(q),
$$

where $I$ denotes a $4 \times 4$ identity matrix. If the geometric compatibility condition (or called the geometric conservation law)

$$
\frac{\partial J}{\partial \tau}-\frac{\partial v}{\partial \xi}=0
$$

is used in the derivation, we have an alternative form of the equations

$$
\frac{\partial(J q)}{\partial \tau}+\frac{\partial}{\partial \xi}(f(q)-v q)+B \frac{\partial q}{\partial \xi}=J \mu \psi(q),
$$

that are essential to many existing moving mesh methods (cf. [?] and references therein).

\section{Relaxation solver on moving meshes}

In each time step, our numerical method for solving barotropic two-phase flows on a moving mesh consists of the following steps:

(1) (Moving mesh step) Determine the cell-interface velocity and the cell-interface location in the physical space over a time step.

(2) (Frozen step $\mu \rightarrow 0$ ) Solve the homogeneous part of the relaxation model described in Section ?? on a moving mapped grid over the same time step as in step 1.

(3) (Relaxation step $\mu \rightarrow \infty$ ) Solve the model system with only the source terms in the infinite relaxation limit. 

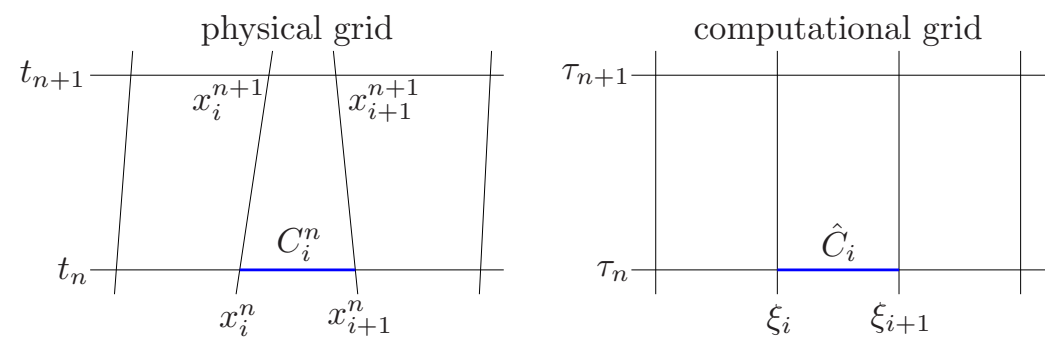

Figure 3.1: A sample grid system in our one-dimensional moving mesh method. The motion of the cell-interface location $x_{i}^{n}$ at a time $t_{n}$ is assumed to move at a constant speed $v_{i}^{n}$ over each time step $\left[t_{n}, t_{n+1}\right]$.

Note that, in step 1, we may use a variational approach as proposed by Tang and Tang [?] for the determination of the cell-interface location from the current time at $x_{i}^{n}$ to the next at $x_{i}^{n+1}$. With that, if the motion of $x_{i}^{n}$ is assumed to move at a constant speed over each time interval $\left[t_{n}, t_{n+1}\right]$, the cell-interface velocity can be computed by $v_{i}^{n}=$ $\left(x_{i}^{n+1}-x_{i}^{n}\right) / \Delta t$, where $\Delta t=t_{n+1}-t_{n}$, see Fig. ?? for an illustration.

It is clear that, alternatively, we may also use a classical Lagrangian approach in this moving-mesh step in that $v_{i}^{n}$ is taken simply as the particle velocity of the local Riemann problem at the cell interface $x_{i}^{n}$. We then set $x_{i}^{n+1}$ to be $x_{i}^{n+1}=x_{i}^{n}+v_{i}^{n} \Delta t$ over a time step $\Delta t$.

Next, in step 2 of the method, we use a finite-volume formulation of the solution in that the approximate value of the cell average of the solution $q$ over the $i$ th grid cell at a time $t_{n}$ can be written as

$$
Q_{i}^{n} \approx \frac{1}{\mathcal{M}\left(\mathcal{C}_{i}^{n}\right)} \int_{\mathcal{C}_{i}^{n}} q\left(x, t_{n}\right) d x=\frac{1}{J_{i}^{n} \Delta \xi} \int_{\hat{\mathcal{C}}_{i}} q\left(\xi, \tau_{n}\right) d \xi
$$

where $\mathcal{C}_{i}^{n}$ and $\hat{\mathcal{C}}_{i}$ represent the regions occupied by the $i$ th grid cell in the physical and computational domains, respectively, and $\mathcal{M}\left(\mathcal{C}_{i}^{n}\right)=x_{i+1}^{n}-$ $x_{i}^{n}=J_{i}^{n} \Delta \xi$ is the measure (length) of $\mathcal{C}_{i}^{n}$. Here we define $J_{i}^{n}=J\left(\mathcal{C}_{i}^{n}\right)$ as being the metric of the mapping of this cell, and assume a uniform mesh size $\Delta \xi=\xi_{i+1}-\xi_{i}$ in the computational domain; the time step from the current time $\tau_{n}$ to the next $\tau_{n+1}$ is denoted by $\Delta \tau=\Delta t$.

In this setup, the first order version of the wave propagation method for the equations (??) without the source terms is a Godunov-type scheme on a mapped grid that can be written in the fluctuation form as

$$
J_{i}^{n+1} Q_{i}^{n+1}=J_{i}^{n} Q_{i}^{n}-\frac{\Delta \tau}{\Delta \xi}\left(\mathcal{A}^{+} \Delta Q_{i}+\mathcal{A}^{-} \Delta Q_{i+1}\right)
$$


where $\mathcal{A}^{+} \Delta Q_{i}$ and $\mathcal{A}^{-} \Delta Q_{i+1}$ are the right- and left-moving fluctuations, respectively, which are defined as

$$
\begin{aligned}
\mathcal{A}^{+} \Delta Q_{i} & =f\left(Q_{i}^{n}\right)-f\left(Q_{i}^{*}\right)+v_{i}^{n} Q_{i}^{*}+B_{i}^{+}\left(Q_{i}^{n}-Q_{i-1}^{n}\right), \\
\mathcal{A}^{-} \Delta Q_{i+1} & =f\left(Q_{i+1}^{*}\right)-f\left(Q_{i}^{n}\right)-v_{i+1}^{n} Q_{i+1}^{*}+B_{i+1}^{-}\left(Q_{i+1}^{n}-Q_{i}^{n}\right) .
\end{aligned}
$$

Here the values $Q_{\iota}^{*}$ for $\iota=i, i+1$ are assigned to be the state obtained in the solution of the Riemann problem between the grid cells with $Q_{\iota-1}^{n}$ and $Q_{\iota}^{n}$ along the ray $\left(x-x_{\iota}^{n}\right) /\left(t-t_{n}\right)=v_{\iota}^{n}$ (cf. [?]). As usual, we set $B^{+}=\max \{B, 0\}$ and $B^{-}=\min \{B, 0\}$.

Note that, to ensure the free-stream (constant states) preservation, which is one of the basic properties that a moving mesh method should have, it is necessary to supplement the method with a consistent discretization of the geometric compatibility condition (??) as

$$
J_{i}^{n+1}=J_{i}^{n}-\frac{\Delta \tau}{\Delta \xi}\left(v_{i}^{n}-v_{i+1}^{n}\right)
$$

for $J_{i}^{n+1}$ with all $i$ and $n$. In one dimension, it is easy to see that (??) is the direct consequence of step 1 for the cell-interface motion. In more than one dimension, however, it requires a careful numerical discretization of the method on a 2D quadrilateral or 3D hexahedral mesh (cf. [?]).

For practical purposes, it is desirable to write the method that retains the free-stream preservation in any space dimension, when the associated geometric conservation law is satisfied only approximately. One approach to do this is discussed in [?] for one-dimensional hyperbolic balance laws in that (??) is substituted to (??), yielding easily

$$
Q_{i}^{n+1}=Q_{i}^{n}-\frac{1}{J_{i}^{n+1}} \frac{\Delta \tau}{\Delta \xi}\left(\mathcal{A}^{+} \Delta Q_{i}+\mathcal{A}^{-} \Delta Q_{i+1}\right)
$$

with

$$
\begin{aligned}
\mathcal{A}^{+} \Delta Q_{i} & =f\left(Q_{i}^{n}\right)-f\left(Q_{i}^{*}\right)-v_{i}^{n}\left(Q_{i}^{n}-Q_{i}^{*}\right)+B_{i}^{+}\left(Q_{i}^{n}-Q_{i-1}^{n}\right), \\
\mathcal{A}^{-} \Delta Q_{i+1} & =f\left(Q_{i+1}^{*}\right)-f\left(Q_{i}^{n}\right)-v_{i+1}^{n}\left(Q_{i+1}^{*}-Q_{i}^{n}\right)+B_{i+1}^{-}\left(Q_{i+1}^{n}-Q_{i}^{n}\right),
\end{aligned}
$$

that is a consistent discretization of equations (??). To achieve high resolution, it is a standard matter to include correction terms to the method, see [?] for the details.

Finally, in step 3, using the solution obtained in step 2 as the initial condition, we solve the model system with the source term of the form

$$
\frac{\partial(J q)}{\partial \tau}=J \mu \psi(q)
$$

which, in the infinite relaxation limit, leads to

$$
g\left(\alpha_{1}^{n+1}\right)=p_{1}\left(\frac{\left(\alpha_{1} \rho_{1}\right)^{n+1}}{\alpha_{1}^{n+1}}\right)-p_{2}\left(\frac{\left(\alpha_{2} \rho_{2}\right)^{n+1}}{1-\alpha_{1}^{n+1}}\right)=0
$$


for the relaxed volume fraction $\alpha_{1}^{n+1}$ which can be solved by a standard iterative root-finding solver such as the secant method.

\section{Numerical results}

We now present preliminary results obtained using our moving mesh method described in Section ?? for barotropic two-phase flow problems with and without cavitation in one space dimension. For simplicity, we assume that the constitutive law for each of the fluid phases satisfies the Tait equation of state of the form

$$
p_{k}(\rho)=\left(p_{0 k}+\mathcal{B}_{k}\right)\left(\frac{\rho}{\rho_{0 k}}\right)^{\gamma_{k}}-\mathcal{B}_{k} \quad \text { for } k=1,2 .
$$

The set of parameters we take in the tests considered here are, for phase 1 (the liquid phase), $\left(\gamma, \mathcal{B}, \rho_{0}, p_{0}\right)_{1}=\left(7,3000 \mathrm{bar}, 10^{3} \mathrm{~kg} / \mathrm{m}^{3}, 1 \mathrm{bar}\right)$, and for phase 2 (the gas phase), $\left(\gamma, \mathcal{B}, \rho_{0}, p_{0}\right)_{2}=\left(1.4,0,1 \mathrm{~kg} / \mathrm{m}^{3}, 1 \mathrm{bar}\right)$. In carrying out the runs, the basic grid motion is governed by the quasiLagrangian approach as $\partial x / \partial \tau=(1-h) u$ with $h=10^{-6}$, and the high resolution version of the method is used throughout.

EXAMPlE ??.1. Our first example is a cavitation-free two-phase (gas-liquid) Riemann problem. Initially, in a region where $x \in[-2,0) m$, we have the state variables

$$
\left(\rho_{1}, \rho_{2}, u, \alpha_{1}\right)_{L}=\left(10^{3} \mathrm{~kg} / \mathrm{m}^{3}, 719.686 \mathrm{~kg} / \mathrm{m}^{3}, 0, \epsilon\right),
$$

while in a region where $x \in[0,2] m$, we have the state variables

$$
\left(\rho 1, \rho_{2}, u, \alpha_{1}\right)_{R}=\left(10^{3} \mathrm{~kg} / \mathrm{m}^{3}, 1 \mathrm{~kg} / \mathrm{m}^{3}, 0,1-\epsilon\right),
$$

where $\epsilon=10^{-6}$.

Figure ?? shows contours of the computed density and pressure in the $x$ - $t$ plane up to time $t=4 \mathrm{~ms}$ using 100 mesh points, where solid wall boundary conditions are used at the both end. It is clear that breaking of the gas-liquid membrane results in a self-similar solution that consists of a left-moving rarefaction wave, a right-moving contact discontinuity, and a shock wave. Complicated wave interactions occur when the waves are reflecting back from the boundaries. From the figure, sharpness of the density and also free of spurious oscillation in the pressure near the contact discontinuity are easily seen. The physical grid system obtained by the method is shown in Fig. ??.

EXAMPLE ??.2. We are next concerned with a water-vapor cavitation problem in that inside a shock tube of one-meter length with 

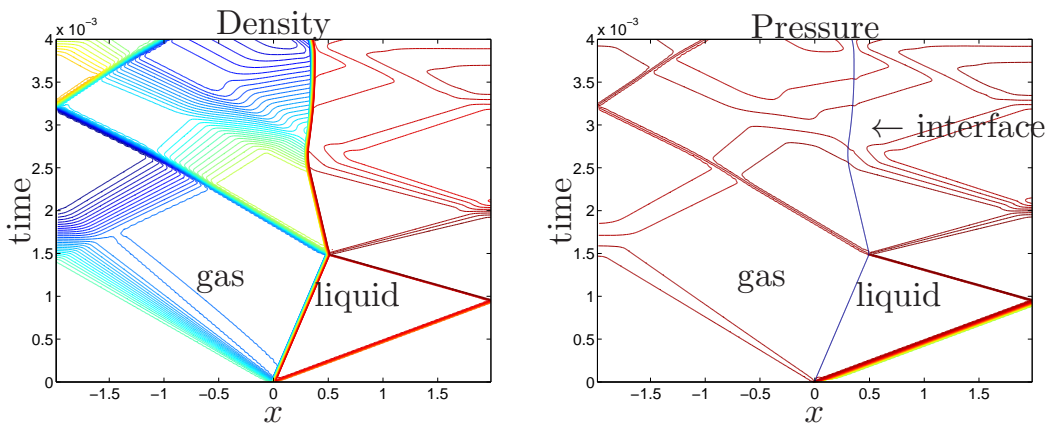

Figure 4.1: Moving mesh results for a two-phase Riemann problem. Contours of the density and pressure are shown in the $x-t$ plane up to $4 \mathrm{~ms}$.
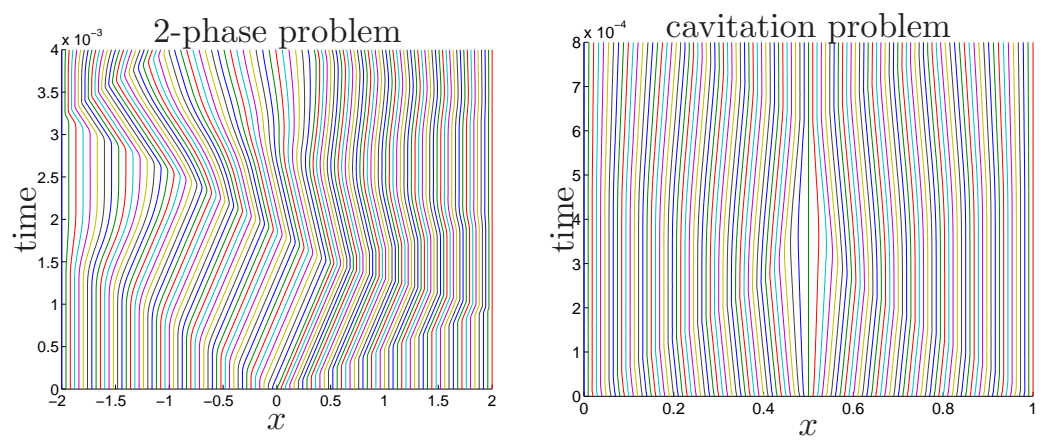

Figure 4.2: Moving meshes in the $x$ - $t$ plane for the two-phase Riemann problem (on the left) and cavitation problem (on the right).

closed ends the fluid is a homogeneous air-water mixture at the standard atmospheric condition $\left(\rho_{1}, \rho_{2}, \alpha_{1}\right)=\left(10^{3} \mathrm{~kg} / \mathrm{m}^{3}, 1 \mathrm{~kg} / \mathrm{m}^{3}, 0,1-\epsilon\right)$, where $\epsilon=10^{-6}$. Initially, inside the tube, there is a jump on the velocity at $x=1 / 2 \mathrm{~m}$ with speed $u=-100 \mathrm{~m} / \mathrm{s}$ on the left and $u=100 \mathrm{~m} / \mathrm{s}$ on the right of the tube. In addition to that, since it is a closed tube, there are jumps also on the velocity at the both ends.

With this condition, as times go on, two rarefaction waves are formed, causing the decrease of the pressure and the formation of the cavitation zone inside the tube. In the meantime, there are inward-moving shock waves propagating from the boundaries, yielding the collapse of the region of cavitation due to the shock-cavitation interaction. Figure ?? shows pseudo-colors of the volume fraction and pressure in the $x$ - $t$ plane up to time $0.8 \mathrm{~ms}$, see Fig. ?? for the meshes over time. We observe reasonable resolution of the results. 

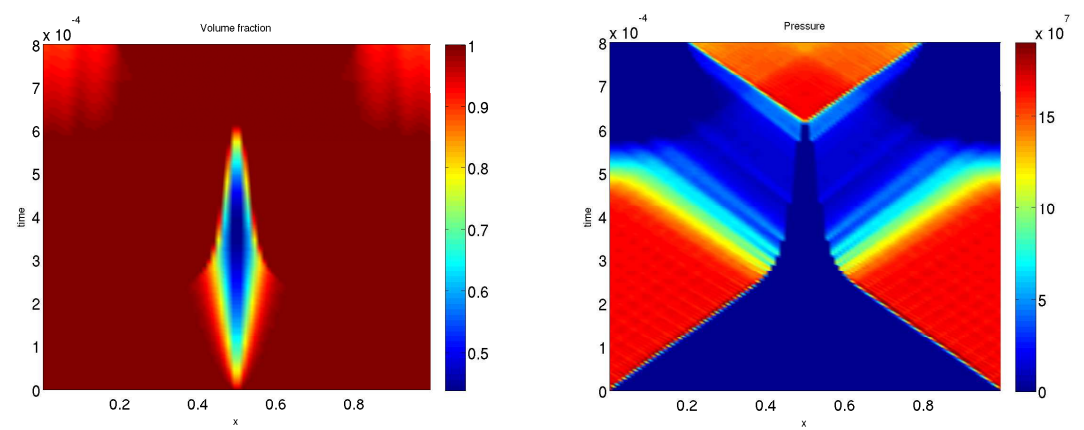

Figure 4.3: Moving mesh results for a water-vapor cavitation problem. Pseudo-colors of the volume fraction and pressure are shown in the $x$ - $t$ plane up to time $0.8 \mathrm{~ms}$.

\section{References}

[1] R. Fazio and R. J. LeVeque. Moving-mesh methods for onedimensional hyperbolic problems using CLAWPACK. Comput. Math. with Appl., 45:273-298, 2003.

[2] W. H. Hui. The unified coordinate system in computational fluid dynamics. Comm. Comput. Phys., 2:577-610, 2007.

[3] F. Petitpas, J. Massoni, R. Saurel, E. Lapebie, and L. Munier. Diffuse interface model for high speed cavitating underwater systems. Int. J. Multiphase Flow, 35:747-759, 2009.

[4] R. Saurel, F. Petitpas, and R. Abgrall. Modelling phase transition in metastable liquids: application to cavitating and flashing flows. $J$. Fluid. Mech., 607:313-350, 2008.

[5] R. Saurel, F. Petitpas, and R. A. Berry. Simple and efficient relaxation methods for interfaces separating compressible fluids, cavitating flows and shocks in multiphase mixtures. J. Comput. Phys., 228:1678-1712, 2009.

[6] H. Tang and T. Tang. Adaptive mesh methods for one- and twodimensional hyperbolic conservation laws. SIAM J. Numer. Anal., 41:487-515, 2003.

[7] P. D. Thomas and C. K. Lombard. Geometric conservation law and its application to flow computations on moving grids. AIAA J., 17:1030-1037, 1979.

[8] S. Venkateswaran, J. W. Lindau, R. F. Kunz, and C. L. Merkle. Computation of multiphase mixture flows with compressibility effects. $J$. Comput. Phys., 180:54-77, 2002. 